\title{
Amelioration Effect of Soil Amendment on Growth and Yield of Vigna unguiculata L. Grown in Acidic and Alkaline Soils
}

\author{
A. A. J. Mofunanya ${ }^{1^{*}}$, E. A. Effa ${ }^{1}$ and I. R. Udosen ${ }^{2}$ \\ ${ }^{1}$ Department of Botany, Faculty of Biological Sciences, University of Calabar, Calabar, Nigeria. \\ ${ }^{2}$ Department of Biology, College of Education, Afaha Nsit, Akwa Ibom State, Nigeria.
}

Authors' contributions

This work was carried out in collaboration among all authors. Author AAJM designed the study, performed the statistical analysis, wrote the protocol, and wrote the first draft of the manuscript. Authors EAE and IRU managed the analyses of the study. Author EAE managed the literature searches All authors read and approved the final manuscript.

Article Information

DOI: $10.9734 / A R R B / 2021 / v 36 i 630390$

Editor(s):

(1) Prof. Tunira Bhadauria, Kanpur University, India.

Reviewers:

(1) S. S. Kushwah, RVS Krishi Vishwa Vidyalaya, India.

(2) Bilal Ahmad Lone, Skuast-K, India.

Complete Peer review History: http://www.sdiarticle4.com/review-history/70709

Original Research Article

Received 20 April 2021

Accepted 27 June 2021

Published 28 June 2021

\section{ABSTRACT}

Aim: Amelioration effect of soil amendment on growth and yield of Vigna unguiculata L. grown in acidic and alkaline soils.

Study Design: The study was conducted in a randomized complete block design with five replicates.

Place and Duration of Study: Department of Botany, University of Calabar, Nigeria, between May and September, 2017.

Methodology: Composite soils from two locations Akamkpa ( $\mathrm{pH} \mathrm{4.0)}$ and Odukpani ( $\mathrm{pH}$ 9.0) were collected, mixed thoroughly with organic manure $(\mathrm{OM})$ and agricultural lime $(\mathrm{AL})$ singly and in combination, allowed to stay three weeks for proper blending before $V$. unguiculata seeds were sown. On seedlings germination, amelioration effect of soil amendment on growth and yield of cowpea grown in acidic and alkaline soils was studied at different periods of growth.

Results: Results revealed significant $(P=0.05)$ increase in leaf area, plant height, number of leaves, leaf fresh and leaf dry weight, relative growth rate, net assimilation rate and leaf area ratio 
of $V$. unguiculata grown in acidic and alkaline soils treated with OM and AL. Pod length, number of pods plant ${ }^{-1}$, number of seeds pod $^{-1}$, number of seeds plant $^{-1}$, weight of 100 seeds increased significantly. Organic manure used singly and in combination was more effective in ameliorating soil $\mathrm{pH}$ effect than AL. Vigna unguiculata performed better in treated and untreated alkaline than in acidic soils. Least plant height for $V$. unguiculata grown in untreated alkaline soil at 12 weeks after sowing WAS was $60.22 \pm 0.16^{\mathrm{a}} \mathrm{cm}$ and highest $119.63 \mathrm{E} 2 \pm .35^{\mathrm{g}} \mathrm{cm}$ for plants grown in soil treated with $\mathrm{AL}_{1}+\mathrm{OM}_{1}$. Least number of seeds plant ${ }^{-1}$ for plants grown in untreated acidic soil at $12 \mathrm{WAS}$ was $90.60 \pm 0.02^{\mathrm{a}}$ and the highest $139.18 \mathrm{E} 2 \pm 0.02^{\mathrm{e}}$ for plants grown in soil treated with $\mathrm{AL}_{1}+\mathrm{OM}_{1}$.

Conclusion: An improvement in growth and seed yield of $V$. unguiculata grown in acidic and alkaline soils is feasible through the use of organic manure and agricultural lime.

Keywords: Acidic soil; agricultural lime; alkaline soil; organic manure; Vigna unguiculata.

\section{INTRODUCTION}

The cowpea (Vigna unguiculata (L.) Walp) is an annual herbaceous legume, well-adapted and widely cultivated in Africa particularly Nigeria. Nigeria is the world's largest producer of $V$. unguiculata. Young leaves, green pods and green seeds of the cowpea are used as vegetable and dry seeds are used in various food preparations [1] for human consumption. The pods and leaves of cowpea are use as fodder for livestock [2]. It also improves soil fertility by atmospheric nitrogen fixation through root nodule rhizobial symbiosis. Vigna unguiculata grains contributes greatly to food and nutrition security in developing countries, especially sub-Saharan Africa, as it is a cheap source of dietary protein complementing the low protein of cereals and tuber crop-based food [3].

The soil quality is very essential for farming and productivity. The need for crop production to meet the food need of the world population is rising. Soil fertility management is critical in assessing food security and poverty alleviation. Soil acidity and alkalinity are serious threats to food production. To overcome the challenge of low crop yield due to soil acidity and alkalinity, crop growers need to optimize nutrient availability to maximize yield. This can be achieved by the application of soil amendments such as organic manure (OM) and agricultural lime (AL). A soil amendment is any kind of material or substrate that is added to the soil in order to improve its physical properties. Soil physical properties that can be amended include soil texture, permeability and water retention. The advantages of soil amendment on crop productivity are multifaceted and include; greater nutrients uptake, greater plant vigor, soil properties improvement [4]. Over the years, soil amendment has been used to improve the nutrient status of soils.

The extent of soil acidity and alkalinity in Cross River State is difficult to quantify. However, reports of acidic soils in various parts of Cross River State are found; in Ogoja [5] in Ikom and Etung [6], in Atimbo and Obufa Esuk [7]. Soils in most parts of Southern Cross River State are acidic with poor yield of crops cultivated on them [8]. In an earlier work, [9] used organic manure and agricultural lime to improve the growth and yield of Phaseolus vulgaris and Vigna aconitifolia grown in acidic, neutral and alkaline soil; also on the net assimilation rate and relative growth rate [10]. No report is found on low yield of $V$. unguiculata grown in soils of the same locations. To remedy low crop productivity for maximum yield, the present study was conducted to assess amelioration effect of soil amendment (organic manure and agricultural lime) on the growth and yield of $V$. unguiculata grown in acidic and alkaline soil of Akamkpa and Odukpani, Southern Cross River State.

\section{MATERIALS AND METHODS}

\subsection{Seeds Collection and Planting}

Vigna unguiculata L. seeds used in this study were obtained from Institute of Agricultural Research and Training Moor Plantation Ibadan, Nigeria. Planting bags from the Ministry of Agriculture, organic manure from the Department of Soil Science, Faculty of Agriculture, University of Calabar and Agricultural lime from Cross River Agricultural Development Project, Calabar. The study was conducted in the Department of Botany Greenhouse, University of Calabar with an average temperature of $25 \pm 3^{\circ} \mathrm{C}$ Calabar. 


\subsection{Soil Collection and pH Determination}

Composite soil samples at depths of $0-20 \mathrm{~cm}$ were collected with the aid of a soil auger from two Local Government Areas of Southern Cross River State. Acidic soil samples ( $\mathrm{pH} 4.0)$ were collected from three villages (Oban, Old Netim and Awi) in Akamkpa LGA. Alkaline soil samples $(\mathrm{pH}$ 9.0) were collected from three villages in Odukpani LGA (Akpan 18 Community, AkimAkim and Okoyong-Usang Abasi). Ten grams each of air-dried composite soil samples was placed in a $100 \mathrm{ml}$ beaker and $50 \mathrm{ml}$ of distilled water added, stirred vigorously with a glass rod for 20 minutes and allowed to stand for 30 minutes. After calibrating the $\mathrm{pH}$ meter with a buffer solution of $\mathrm{pH} 4.0$ and 9.0 , the $\mathrm{pH}$ was read by immersing the electrode into the upper part of the mixture suspension [11].

\subsection{Planting Procedure and Treatment Application}

Eighty four planting bags $(16 \mathrm{~cm}$ internal diameter) were filled with $5 \mathrm{~kg}$ of composite acidic and alkaline soil. These were divided into two groups of 42 planting bags for each soil type. The bags were arranged in a randomized complete block design (RCBD) with three replicates. The soils were treated with agricultural lime $(A L)$ and organic manure $(O M)$ at $0 \mathrm{~g}, 100 \mathrm{~g}$ and $200 \mathrm{~g}$ respectively and allowed to stay for three weeks to enhance blending and interaction between treatment combinations before seed sowing. Two seeds of $V$. unguiculata were sown in each polybag at a depth of $2 \mathrm{~cm}$, after germination, young seedlings were stalked, watered regularly and grown for 16 weeks.

\subsection{Amelioration Effect of Soil Amendment on Growth Parameters of Vigna unguiculata}

The leaf area of $V$. unguiculata grown in acidic and alkaline soils treated with organic and agricultural lime was determined by measuring the length of the leaf in centimeter square and breadth and calculated by the equation [12].

$$
\mathrm{A} f \mathrm{LW}=27.7418+(3.9812 \mathrm{LW} / \mathrm{IN} \text { LW })
$$

Where $L$ is leaf length and $W$ is leaf width

The effect of organic manure and agricultural lime on the shoot height of $V$.unguiculata grown in acidic and alkaline soil was measured using a tape rule in $(\mathrm{cm})$ from the soil level to the terminal bud. Number of leaves per plant of $V$. unguiculata grown in acidic soil treated with organic manure and agricultural lime were counted. These measurements were taken at an initial period of 2 weeks after sowing (WAS), and at 4 weeks, subsequently at 4 weeks intervals to ascertain the amelioration effect of soil amendment on number of leaves produced.

Effect of amelioration on the relative growth rate (RGR)which measures the change in dry weight of different samples of $V$. unguiculata grown on acidic and alkaline soil treated with different grams $(0,100$, and $200 \mathrm{~g})$ of organic manure and agriculture lime. The differences were calculated between plants grown on treated acidic and alkaline soils and untreated soils as described by [13] and the dry weight of whole plant was used in the determination of RGR.

Where

$$
R G R=\frac{\left(\text { In W }_{2}-\mathrm{In} \mathrm{W}_{1}\right)}{\mathrm{t}_{2}-\mathrm{t}_{1}}\left(\mathrm{gg}^{-1} \mathrm{Wk}^{-1}\right)
$$

In = Natural logarithm

$\mathrm{W}_{1}=$ Initial dry weight

$\mathrm{W}_{2}=$ Final dry weight

$t_{1}=$ Initial time

$t_{2}=$ Final

$\mathrm{t}_{1}-\mathrm{t}_{2}=$ Time interval during which time biomass increased from $W_{1}-W_{2}$

Net assimilation rate (NAR) is a function of the photosynthetic effectiveness of leaves of a plant and its leafiness. Net assimilation rate was calculated as the change in total plant biomass per leaf weight and time [14]. Time was used to show the photosynthetic effectiveness $V$. unguiculata grown on acidic and alkaline soils treated with organic manure and agricultural lime.

$$
\mathrm{NAR}=\left(\frac{\mathrm{InA}_{2}-\operatorname{InA} A_{1}}{A_{2}-A_{1}}\right)\left(\frac{\mathrm{W}_{2}-\mathrm{W}_{1}}{\mathrm{t}_{2}-\mathrm{t}_{1}}\right)\left(g g^{-1} \mathrm{Wk}^{-1}\right)
$$

Where

In = Natural logarithm

$\mathrm{A}_{1}=$ Initial leaf area

$A_{2}=$ Subsequent leaf area

$\mathrm{W}_{1}=$ Initial leaf dry weight

$W_{2}=$ Subsequent leaf dry weight

$\mathrm{t}_{1}=$ Initial growth period

$t_{2}=$ Subsequent growth period 
Leaf area ratio (LAR) of $V$. unguiculata grown in treated and untreated soils represents the ratio of total leaf area to whole leaf dry weight over a period of time (4 weeks interval) calculated by the formula given by [15].

$$
\begin{aligned}
& \mathrm{LAR}=\underline{\mathrm{A}}_{1}+\underline{\mathrm{A}_{2}}+\ldots \underline{\mathrm{An}}\left(\mathrm{cm}^{2} \mathrm{~g}^{-1}\right) \\
& W_{1}+W_{2} W n
\end{aligned}
$$

Where

$\mathrm{A}_{1}=$ Leaf area

$A_{2}=$ Subsequent leaf area

$A_{n}=$ Leaf area at $n$...number of times

$W_{1}=$ Initial leaf dry weight

$W_{2}=$ Subsequent leaf dry weight

$\mathrm{W}_{\mathrm{n}}=$ Leaf dry weight at $\mathrm{n}$.....number of times

\subsection{Amelioration Effect of Soil Amendment on Yield Components of Vigna unguiculata}

Dry pods of $V$. unguiculata at maturity were harvested from plants grown in acidic and alkaline soil treated with different concentrations of organic manure and agricultural lime. Number of pods per plant, number of seeds per pod and number of seeds per plant were obtained by counting. The weight of pod and weight of 100 seeds were weighed using analytical weighing balance. Pod length, seed length, pod width and seed width were determined by measuring.

\subsection{Statistical Analysis}

Data analysis was done using LSD test on SPSS version 23. The data presented are mean of five replicates \pm standard deviation. The differences in mean were considered significant at $\mathrm{P}=0.05$ when treatments mean compared to the untreated.

\section{RESULTS}

\subsection{Amelioration Effect of Soil Amendment on Plant Height (cm plant $^{-1}$ ) of Vigna unguiculata Grown in Acidic and Alkaline Soil}

Plant height of $V$. unguiculata grown in acidic and alkaline soils of Akamkpa and Odukpani was significantly $(P=0.05)$ improved when soils were treated with soil amendment. Height of the plant grown in treated soils showed significant $(P=0.05)$ increase from 2 to 12 WAS, plant height did not differ at 16 WAS. Organic manure and agricultural lime used singly $\left(O M_{1}, O M_{2}, A L_{1}\right.$,
$\left.\mathrm{AL}_{2}\right)$ and in combination $\left(\mathrm{OM}_{1}+\mathrm{AL}_{1}\right.$ and $\mathrm{OM}_{2}+$ $A L_{2}$ ) impacted positively on $V$. unguiculata's plant height. The least plant height at different stages of growth was obtained for cowpea grown in untreated acidic soil. Cowpea grown in untreated and treated alkaline soil had higher plant heights than plants grown in acidic soil. Untreated acidic and alkaline soils had the least plant height of $19.71 \pm 0.08^{\mathrm{a}}, 20.89 \pm 0.89^{\mathrm{a}} \mathrm{cm}$ and $46.41 \pm 0.36^{\mathrm{a}}$, $60.22 \pm 0.16^{a} \mathrm{~cm}^{\mathrm{c}}$ plant $^{-1}$ at 2 and 12 WAS. Acidic and alkaline soils treated singly with different concentrations of soil amendment $(0,100,200 \mathrm{~g})$ and in combination had higher plants. Plant height at 2 and 12 WAS for soil treated with 100 $g$ combined $\mathrm{AL}_{1}+\mathrm{OM}_{1}$ were 22.51 $\pm 0.15^{\mathrm{d}}$, $22.91 \pm 0.44^{\mathrm{C}} \mathrm{cm}$ and $92.47 \pm 0.06^{\mathrm{de}}$, 119.63E $2 \pm .35^{\mathrm{g}} \mathrm{cm}$ (Table 1).

\subsection{Amelioration Effect of soil Amendment on Leaf Area $\left(\mathrm{cm}^{2}\right.$ plant $\left.{ }^{-1}\right)$ of Vigna unguiculata Grown in Acidic and Alkaline Soils}

Acidic and alkaline soils of Akamkpa and Odukpani treated with organic manure and agricultural lime used singly and in combination significantly $(P=0.05)$ improved the leaf area of $V$ unguiculata when compared to the untreated soils. At all periods of growth the leaf area of the cowpea grown in untreated soils was significantly lower than that grown in treated soils. Alkaline soil treated with a combination of $\mathrm{AL}_{2}+\mathrm{OM}_{2}$ had the highest leaf area of $36.57 \pm 0.02^{\mathrm{g}}$ at 2 WAS, at 4, 8, 12 and 16 WAP, plants grown in $\mathrm{AL}_{1}+\mathrm{OM}_{1}$ had highest values of $68.37 \pm 0.03^{\mathrm{C}} \mathrm{cm}^{2}$, $81.35 \pm 0.05^{\mathrm{e}}$ and $82.04 \pm 0.02^{\mathrm{f}} \mathrm{cm}^{2}$ at 16 WAS. Akamkpa soil with $\mathrm{pH} 4.0$ when treated with combination of $\mathrm{OM}_{1}+\mathrm{AL}_{1}$ caused increase in leaf size of $29.64 \pm 0.02^{\mathrm{g}}, \quad 46.28 \pm 0.06^{\mathrm{g}}$, $59.82 \pm 0.02^{\mathrm{g}}$ and $66.03 \pm 0.03^{\mathrm{e}} \mathrm{cm}^{2}$ at $2,4,8$ and 12 WAS respectively compared to least values $20.39 \pm 0.03^{\mathrm{a}}, \quad 24.47 \pm 0.04^{\mathrm{a}}, \quad 40.91 \pm 0.05^{\mathrm{a}}$ and $41.06 \pm 0.06^{a}$ respectively. At 16 WAS leaf area did not differ statistically from value for 12 WAS for untreated soils. Acidic and alkaline soils treated with $\mathrm{OM}$ and $\mathrm{AL}$ at various concentrations increased leaf size of $V$. unguiculata when compared to untreated soil types (Table 2).

\subsection{Amelioration Effect of Soil Amendment on Number of Leaves Plant $^{-1}$ of Vigna unguiculata Grown in Acidic and Alkaline Soil}

Results revealed significant $(P=0.05)$ increase in number of leaves produced per of $V$. unguiculata 
grown in treated soils compared to untreated soils. At 2 WAS there was no significant difference in number of leaves produced. However, the number of leaves produced by plants grown in acidic and alkaline soils treated with $\mathrm{OM}$ and $\mathrm{AL}$ singly and in combination increase significantly from 4 to 12 WAS. Untreated acidic soil had the least significant number of leaves per plant of $2.02 \pm 0.006^{\mathrm{a}}$ and $10.53 \pm 0.03^{\mathrm{a}}$ at 2 WAS and 12 WAS as against the highest treated soil values of $2.90 \pm 1.00^{\mathrm{b}}$ $\left(\mathrm{AL}_{1}+\mathrm{OM}_{1}\right)$ at 2 WAS and 20.18 $\pm 0.01^{\mathrm{d}}$ $\left(\mathrm{AL}_{2}+\mathrm{OM}_{2}\right)$ at $12 \mathrm{WAS}$. Corresponding number of leaves for alkaline soil treated with $\mathrm{AL}_{1}+\mathrm{OM}_{1}$ were $3.03 \pm 0.01^{\mathrm{e}}$ at $2 \mathrm{WAS}$ and $38.11 \pm 0.04^{\mathrm{g}}$ at 12 WAS compared to untreated alkaline soil values of $2.18 \pm 0.02^{\mathrm{a}}$ and $15.52 \pm 0.04^{\mathrm{a}}$ (Table 3 ).

\subsection{Amelioration Effect of Soil Amendment on Leaf Fresh Weight (g plant $^{-1}$ ) of Vigna unguiculata Grown in Acidic and Alkaline Soil}

There was significant $(P=0.05)$ variation in leaf fresh weight of plants grown in acidic and alkaline soils treated with OM and Al compared to plants grown in untreated soil (Table 4). The least LFW for untreated acidic soil at 2 and 12 WAS were $1.23 \pm 0.03^{\mathrm{a}}$ and $8.67 \pm 0.03^{\mathrm{a}} \mathrm{g}$ and highest $L F W$ for soil treated $A L_{1}+\mathrm{OM}_{1}$ were $1.62 \pm 0.01^{\mathrm{d}}$ and $24.51 \pm 1.02^{\mathrm{d}} \mathrm{g}$. The LFW of $V$. unguiculata grown in alkaline soil treated with $\mathrm{AL}_{1}+\mathrm{OM}_{1}$ at 2 WAS and 12 WAS were the highest with values of $2.02 \pm 0.02^{\mathrm{d}}$ and $36.03 \pm 0.03^{f} \mathrm{~g}$ as against least LFW of untreated soil of $1.27 \pm 0.03^{\mathrm{a}}$ and $10.40 \pm 0.08^{\mathrm{a}} \mathrm{g}$.

\subsection{Amelioration Effect of Soil Amendment on Leaf Dry Weight (g plant $^{-1}$ ) of Vigna unguiculata Grown on Acidic and Alkaline Soil}

Results in Table 5 showed increase in LDW of $V$. unguiculata grown in acid and alkaline soils treated with different concentrations of OM and AL. Plants grown in untreated acid and alkaline soils had the least significant LDW per plant of $0.24 \pm 0.02^{\mathrm{a}}, 1.56 \pm 0.18^{\mathrm{a}}, 2.01 \pm 0.01^{\mathrm{a}}$, $5.14 \pm 0.01^{\mathrm{a}} \mathrm{g}$ and $5.14 \pm 0.01^{\mathrm{a}}, 1.95 \pm 0.02^{\mathrm{a}}$, $2.87 \pm 0.59^{\mathrm{a}}, 6.71 \pm 0.01^{\mathrm{a}} \mathrm{g}$ at $2,4,8$ and $12 \mathrm{WAS}$ respectively. Highest LDW for acid and alkaline soils treated with $\mathrm{AL}_{1}+\mathrm{OM}_{1}$ at 2, 4, 8 and 12 WAS were $0.27 \pm 0.01^{\mathrm{a}}, 3.72 \pm 0.02^{\mathrm{c}}$, $4.80 \pm 0.03^{\mathrm{d}}, \quad 9.43 \pm 0.01^{\mathrm{c}} \mathrm{g}$ and $0.37 \pm 0.02^{\mathrm{b}}$, $4.11 \pm 0.01^{\mathrm{e}}, \quad 10.85 \pm 0.60^{\mathrm{d}}, \quad 19.57 \pm 0.02^{\mathrm{cd}} \quad \mathrm{g}$ respectively. At initial period of growth
(2 WAS) LDW of the cowpea grown in treated soils did not differ from plants grown in untreated soils.

\subsection{Amelioration Effect of Soil Amendment on Relative Growth Rate (RGR) Plant ${ }^{-1}$ of Vigna unguiculata Grown in Acidic and Alkaline Soil}

Results of RGR of $V$. unguiculata grown in acidic and alkaline soils from Akamkpa and Odukpani soils treated with soil amendment differ statistically from the untreated. Cowpea grown in both soils types treated with soil amendment revealed amelioration effect with significant $(P=0.05)$ increase in $R G R$. Relative growth rate of $V$. unguiculata grown in untreated and treated soils decreased with increasing period of growth. Least significant RGR for plant grown in untreated acidic and alkaline soils were at 4 WAS were $0.0071 \pm 0.00^{\mathrm{a}}$ and $0.0097 \pm 0.00^{\mathrm{a}} \mathrm{gg}^{-}$ $\left.{ }^{1} \mathrm{wk}^{-1}\right)$, at16 WAS were $0.0033 \pm 0.00^{\mathrm{a}}$ and $0.0048 \pm 0.00^{\mathrm{a}}\left(\mathrm{gg}^{-1} \mathrm{wk}^{-1}\right)$. Highest RGR of soils treated with $\mathrm{OM}$ and $\mathrm{AL}$ showed amelioration effect with increase in RGR of plants grown in acidic and alkaline soils of $0.0180 \pm 0.00^{\mathrm{e}}$ and $0.0186 \pm 0.00^{f}\left(\mathrm{gg}^{-1} \mathrm{wk}^{-1}\right)$ as against untreated soil RGR $0.0071 \pm 0.00^{\mathrm{a}}$ and $0.0097 \pm 0.00^{\mathrm{a}}\left(\mathrm{gg}^{-1} \mathrm{wk}^{-1}\right)$ (Table 6).

\subsection{Amelioration Effect of Soil Amendment on Net Assimilation Rate of Vigna unguiculata Grown in Acidic and Alkaline Soil}

Amelioration effect of soil amendment led to increase in NAR. Results of NAR of $V$. unguiculata grown in soils from Akamkpa and Odukpani treated with organic manure and agricultural lime are presented in Table 7. At 4 WAS, results revealed significant $(P=0.05)$ increase in NAR of plants grown in the two soil types. The highest NAR was obtained for $V$. unguiculata grown in acidic soil treated with $A L_{1}$, $\mathrm{AL}_{2}, \quad \mathrm{OM}_{1}, \quad \mathrm{OM}_{2}, \quad \mathrm{AL} 1+\mathrm{OM}_{1}$ and $\quad \mathrm{AL}_{2}+\mathrm{OM}_{2}$ $\left(0.0010 \pm 0.00^{\mathrm{C}} \quad \mathrm{ggcm}^{-2} \mathrm{wk}^{-1}\right)$ compared to untreated soil NAR of $0.0006 \pm 0.00^{\mathrm{a}}\left(\mathrm{ggcm}^{-2} \mathrm{wk}^{-1}\right)$. Alkaline soil of Odukpani treated with $\mathrm{AL}_{2}+\mathrm{OM}_{2}$ had the highest NAR of $0.0018 \pm 0.00^{\mathrm{d}}\left(\mathrm{ggcm}^{-2} \mathrm{wk}^{-}\right.$ $\left.{ }^{1}\right)$ as against untreated NAR of $0.0009 \pm 0.00^{\mathrm{a}}$ $\left(\mathrm{ggcm}^{-2} \mathrm{Wk}^{-1}\right)$ at 4 WAS. At 12 WAS, acidic soil treated with $\mathrm{AL}_{1}+\mathrm{OM}_{1}$ and $\mathrm{AL}_{2}+\mathrm{OM}_{2}$ had the highest NAR of $0.00081 \pm 0.00^{\mathrm{C}}\left(\mathrm{ggcm}^{-2} \mathrm{wk}^{-1}\right)$ compared untreated acidic soil NAR of $0.0004 \pm 0.00^{\mathrm{a}}\left(\mathrm{ggcm}^{-2} \mathrm{wk}^{-1}\right)$. Least NAR of 
Table 1. Amelioration effect of soil amendment on plant height $\left(\mathrm{cm} \mathrm{plant}^{-1}\right)$ of Vigna unguiculata grown in acidic and alkaline soil

\begin{tabular}{|c|c|c|c|c|c|c|c|c|c|c|}
\hline \multirow{2}{*}{$\begin{array}{l}\text { Weeks } \\
\text { Soil type }\end{array}$} & \multicolumn{2}{|c|}{2 WAS } & \multicolumn{2}{|c|}{4 WAS } & \multicolumn{2}{|c|}{8 WAS } & \multicolumn{2}{|c|}{12 WAS } & \multicolumn{2}{|c|}{16 WAS } \\
\hline & Acidic soil & Alkaline soil & Acidic soil & Alkaline soil & Acidic soil & Alkaline soil & Acidic soil & Alkaline soil & Acidic soil & Alkaline soil \\
\hline Untreated & $19.71 \pm 0.08^{a}$ & $20.89 \pm 0.89^{\mathrm{a}}$ & $28.71 \pm 0.04^{\mathrm{a}}$ & $41.37 \pm 0.05^{a}$ & $36.66 \pm 0.20^{a}$ & $58.93 \pm 0.06^{\mathrm{a}}$ & $46.41 \pm 0.36^{a}$ & $60.22 \pm 0.16^{a}$ & $49.56 \pm 0.29^{a}$ & $63.05 \pm 0.06^{\mathrm{a}}$ \\
\hline \multicolumn{11}{|c|}{ Treated } \\
\hline $\mathrm{AL}_{1}$ & $20.66 \pm 0.93^{b}$ & $21.65 \pm 0.07^{b}$ & $38.66 \pm 0.19^{b}$ & $44.74 \pm 0.07^{b}$ & $69.39 \pm 0.23^{c}$ & $86.36 \pm 0.23^{\mathrm{e}}$ & $78.25 \pm 0.12^{\mathrm{C}}$ & $90.18 \pm 0.03^{c}$ & $80.62 \pm 0.05^{b}$ & $90.10 \pm 0.04^{b}$ \\
\hline $\mathrm{AL}_{2}$ & $20.37 \pm 0.41^{b}$ & $21.54 \pm 0.45^{\mathrm{b}}$ & $38.31 \pm 0.58^{\mathrm{c}}$ & $45.54 \pm .4 .45^{\mathrm{b}}$ & $63.60 \pm 0.15^{b}$ & $80.26 \pm 0.12^{b}$ & $72.26 \pm 0.13^{b}$ & $88.75 \pm 0.15^{b}$ & $79.11 \pm 0.02^{b}$ & $89.26 \pm 0.40^{c}$ \\
\hline $\mathrm{OM}_{1}$ & $22.43 \pm 0.20^{d}$ & $21.85 \pm 0.55^{\mathrm{b}}$ & $40.44 \pm 0.36^{d}$ & $44.65 \pm 0.2^{b}$ & $77.29 \pm 0.14^{e}$ & $85.52 \pm 0.03^{d}$ & $90.88 \pm 0.15^{d}$ & $99.39 \pm 0.13^{d}$ & $96.14 \pm 4.38^{\mathrm{C}}$ & $99.41 \pm 0.02^{d}$ \\
\hline $\mathrm{OM}_{2}$ & $22.13 \pm 0.77^{d}$ & $23.93 \pm 0.18^{d}$ & $45.48 \pm 0.05^{\mathrm{d}}$ & $45.6740 \pm 0.23^{b}$ & $80.29 \pm 0.19^{f}$ & $90.13 \pm 0.13^{f}$ & $94.57 \pm 0.11^{f}$ & $101.11 \mathrm{E} 2 \pm 1.23^{\mathrm{e}}$ & $94.51 \pm 0.12^{\mathrm{e}}$ & $100.01 \pm 0.79^{\mathrm{e}}$ \\
\hline $\mathrm{AL}_{1}+\mathrm{OM}_{1}$ & $22.51 \pm 0.15^{d}$ & $22.91 \pm 0.44^{c}$ & $40.97 \pm 0.24^{\mathrm{e}}$ & $48.28 \pm .0 .02^{\mathrm{C}}$ & $81.89 \pm 0.03^{9}$ & $90.66 \pm 0.08^{9}$ & $92.47 \pm 0.06^{\mathrm{de}}$ & $119.63 \mathrm{E} 2 \pm .35^{9}$ & $92.49 \pm 0.029^{d}$ & $100.11 \pm 0.37^{f}$ \\
\hline $\mathrm{AL}_{2}+\mathrm{OM}_{2}$ & $21.61 \pm 0.85^{c}$ & $22.79 \pm 0.11^{\mathrm{c}}$ & $40.51 \pm 0.35^{f}$ & $46.60 \pm .0 .01^{c}$ & $75.84 \pm 0.03^{d}$ & $82.72 \pm 0.03^{c}$ & $97.38 \pm 0.02^{9}$ & $110.71 \mathrm{E} 2 \pm 0.20^{f}$ & $97.51 \pm 0.02^{f}$ & $100.20 \pm 0.02^{\mathrm{g}}$ \\
\hline
\end{tabular}

organic manure, $A L_{2}=200 \mathrm{~g}$ agricultural lime, $O M_{2}=200 \mathrm{~g}$ organic manure, $O M_{1}+A L_{1}=50 \mathrm{~g}$ organic manure $+50 \mathrm{~g}$ agricultural lime, $O M_{2}+A L_{2}=100 \mathrm{~g}$ organic manure $+100 \mathrm{~g}$ agricultural lime

Table 2. Amelioration effect of soil amendment on leaf area $\left(\mathrm{cm}^{2}\right.$ plant $\left.{ }^{-1}\right)$ of Vigna unguiculata grown in two soil types

\begin{tabular}{|c|c|c|c|c|c|c|c|c|c|c|}
\hline \multirow{2}{*}{$\begin{array}{l}\text { Weeks } \\
\text { Soil types }\end{array}$} & \multicolumn{2}{|c|}{2 WAS } & \multicolumn{2}{|c|}{4 WAS } & \multicolumn{2}{|c|}{8 WAS } & \multicolumn{2}{|c|}{12 WAS } & \multicolumn{2}{|c|}{16 WAS } \\
\hline & Acidic soil & Alkaline soil & Acidic soil & Alkaline soil & Acidic soil & Alkaline soil & Acidic soil & Alkaline soil & Acidic soil & Alkaline soil \\
\hline Untreated & $20.39 \pm 0.03^{a}$ & $27.97 \pm 0.02^{\mathrm{a}}$ & $24.47 \pm 0.04^{\mathrm{a}}$ & $37.01 \pm 0.01^{a}$ & $40.91 \pm 0.05^{a}$ & $46.71 \pm 0.02^{\mathrm{a}}$ & $41.06 \pm 0.06^{a}$ & $60.08 \pm 0.02^{a}$ & $41.01 \pm 0.03^{\mathrm{a}}$ & $60.10 \pm 0.02^{\mathrm{a}}$ \\
\hline \multicolumn{11}{|c|}{ Treated } \\
\hline $\mathrm{AL}_{1}$ & $22.18 \pm 0.02^{b}$ & $29.68 \pm 0.05^{b}$ & $33.16 \pm 0.01^{\mathrm{c}}$ & $48.26 \pm 0.04^{b}$ & $55.64 \pm 0.03^{\mathrm{d}}$ & $60.19 \pm 0.05^{b}$ & $52.06 \pm 0.04^{b}$ & $73.18 \pm 0.02^{c}$ & $52.04 \pm 0.04^{c}$ & $73.13 \pm 0.03^{d}$ \\
\hline $\mathrm{AL}_{2}$ & $23.40 \pm 0.01^{c}$ & $30.57 \pm 0.03^{d}$ & $30.35 \pm 0.05^{b}$ & $40.60 \pm 0.02^{a}$ & $42.74 \pm 0.02^{b}$ & $63.66 \pm 0.01^{\mathrm{c}}$ & $50.85 \pm 0.03^{b}$ & $77.11 \pm 0.01^{\mathrm{e}}$ & $50.86 \pm 0.02^{b}$ & $70.08 \pm 0.02^{b}$ \\
\hline $\mathrm{OM}_{1}$ & $24.61 \pm 0.02^{d}$ & $34.60 \pm 0.03^{\mathrm{e}}$ & $34.24 \pm 0.05^{d}$ & $65.20 \pm 0.08^{c}$ & $50.01 \pm 0.01^{c}$ & $60.17 \pm 0.03^{b}$ & $54.26 \pm 3.50^{c}$ & $75.81 \pm 0.01^{\mathrm{d}}$ & $56.24 \pm 0.02^{\mathrm{e}}$ & $75.67 \pm 0.01^{\mathrm{e}}$ \\
\hline $\mathrm{OM}_{2}$ & $27.70 \pm 0.10^{\mathrm{e}}$ & $30.35 \pm 0.01^{\mathrm{c}}$ & $40.32 \pm 0.02^{\mathrm{e}}$ & $70.36 \pm 0.01^{c}$ & $58.98 \pm 0.01^{\mathrm{e}}$ & $94.26 \pm 0.03^{f}$ & $54.74 \pm 0.04^{\mathrm{c}}$ & $94.35 \pm 0.05^{9}$ & $54.69 \pm 0.01^{d}$ & $94.28 \pm 0.02^{f}$ \\
\hline $\mathrm{AL}_{1}+\mathrm{OM}_{1}$ & $29.64 \pm 0.02^{9}$ & $35.92 \pm 0.02^{f}$ & $46.28 \pm 0.06^{g}$ & $68.37 \pm 0.03^{c}$ & $59.82 \pm 0.02^{9}$ & $81.35 \pm 0.05^{\mathrm{e}}$ & $66.03 \pm 0.03^{\mathrm{e}}$ & $82.04 \pm 0.02^{f}$ & $66.00 \pm 0.01^{f}$ & $82.02 \pm 0.02^{9}$ \\
\hline $\mathrm{AL}_{2}+\mathrm{OM}_{2}$ & $29.54 \pm 0.03^{f}$ & $36.57 \pm 0.02^{\mathrm{g}}$ & $42.63 \pm 0.03^{f}$ & $53.34 \pm 0.04^{b}$ & $59.18 \pm 0.03^{f}$ & $74.83 \pm 0.18^{\mathrm{d}}$ & $60.20 \pm 0.10^{d}$ & $72.13 \pm 0.07^{b}$ & $60.17 \pm .0 .02^{\mathrm{e}}$ & $72.13 \pm 0.03^{\mathrm{C}}$ \\
\hline
\end{tabular}
organic manure, $A L_{2}=200 \mathrm{~g}$ agricultural lime, $O M_{2}=200 \mathrm{~g}$ organic manure, $O M_{1}+A L_{1}=50 \mathrm{~g}$ organic manure $+50 \mathrm{~g}$ agricultural lime, OM $\mathrm{M}_{2}+A L_{2}=100 \mathrm{~g}$ organic manure $+100 \mathrm{~g}$ agricultural lime 
Table 3. Amelioration effect of soil amendment on number of leaves plant ${ }^{-1}$ of Vigna unguiculata grown in acidic and alkaline soil

\begin{tabular}{|c|c|c|c|c|c|c|c|c|c|c|}
\hline \multirow{2}{*}{$\begin{array}{l}\text { Weeks } \\
\text { Soil type }\end{array}$} & \multicolumn{2}{|c|}{2 WAS } & \multicolumn{2}{|c|}{4 WAS } & \multicolumn{2}{|c|}{8 WAS } & \multicolumn{2}{|c|}{12 WAS } & \multicolumn{2}{|c|}{16 WAS } \\
\hline & Acidic soil & Alkaline soil & Acidic soil & Alkaline soil & Acidic soil & Alkaline soil & Acidic soil & Alkaline soil & Acidic soil & Alkaline soil \\
\hline Untreated & $2.02 \pm 0.006^{a}$ & $2.18 \pm 0.02^{\mathrm{a}}$ & $4.39 \pm 0.03^{\mathrm{a}}$ & $4.83 \pm 0.01^{a}$ & $7.69 \pm 0.03^{a}$ & $4.56 \pm 4.04^{\mathrm{a}}$ & $10.53 \pm 0.03^{\mathrm{a}}$ & $15.52 \pm 0.04^{a}$ & $12.55 \pm 0.01^{\mathrm{a}}$ & $15.49 \pm 0.01^{\mathrm{a}}$ \\
\hline \multicolumn{11}{|c|}{ Treated } \\
\hline $\mathrm{AL}_{1}$ & $2.45 \pm 0.03^{\mathrm{a}}$ & $2.79 \pm 0.03^{c}$ & $6.52 \pm 0.05^{b}$ & $8.01 \pm 0.01^{b}$ & $10.72 \pm 0.01^{\mathrm{b}}$ & $15.38 \pm 0.01^{b}$ & $17.70 \pm 0.04^{b}$ & $22.84 \pm 0.02^{b}$ & $18.53 \pm 0.03^{b}$ & $22.18 \pm 0.02^{b}$ \\
\hline $\mathrm{AL}_{2}$ & $2.62 \pm 0.02^{\mathrm{a}}$ & $2.86 \pm 0.04^{d}$ & $6.44 \pm 0.04^{b}$ & $8.91 \pm 0.05^{d}$ & $11.43 \pm 0.03^{c}$ & $17.65 \pm 0.05^{b}$ & $18.33 \pm 1.00^{b}$ & $24.42 \pm 0.06^{c}$ & $21.96 \pm 1.02^{c}$ & $23.82 \pm 0.02^{d}$ \\
\hline $\mathrm{OM}_{1}$ & $2.77 \pm 0.01^{b}$ & $2.90 \pm 0.01^{\mathrm{d}}$ & $6.50 \pm 0.99^{b}$ & $8.98 \pm 0.01^{\mathrm{e}}$ & $12.28 \pm 0.02^{\mathrm{e}}$ & $18.01 \pm 0.01^{\mathrm{C}}$ & $19.19 \pm 0.03^{c}$ & $29.57 \pm 0.07^{d}$ & $22.38 \pm 0.07^{\mathrm{c}}$ & $29.25 \pm 0.02^{c}$ \\
\hline $\mathrm{OM}_{2}$ & $2.32 \pm 0.02^{\mathrm{a}}$ & $2.53 \pm 0.03^{b}$ & $6.41 \pm 1.00^{\mathrm{b}}$ & $8.74 \pm 0.04^{c}$ & $11.65 \pm 0.05^{\mathrm{d}}$ & $19.22 \pm 0.01^{\mathrm{c}}$ & $19.31 \pm 0.09^{c}$ & $30.45 \pm 0.05^{\mathrm{e}}$ & $22.46 \pm 1.02^{\mathrm{C}}$ & $30.16 \pm 0.02^{\mathrm{e}}$ \\
\hline $\mathrm{AL}_{1}+\mathrm{OM}_{1}$ & $2.90 \pm 1.00^{b}$ & $3.03 \pm 0.01^{\mathrm{e}}$ & $7.20 \pm 0.03^{b}$ & $10.04 \pm 0.02^{g}$ & $11.80 \pm 0.05^{\mathrm{d}}$ & $23.98 \pm 1.00^{d}$ & $20.01 \pm 0.01^{d}$ & $38.11 \pm 0.04^{g}$ & $24.48 \pm 0.0^{d}$ & $38.01 \pm 0.03^{g}$ \\
\hline $\mathrm{AL}_{2}+\mathrm{OM}_{2}$ & $2.88 \pm 0.01^{b}$ & $2.99 \pm 0.01^{\mathrm{e}}$ & $7.41 \pm 0.02^{b}$ & $9.87 \pm 0.01^{\dagger}$ & $11.76 \pm 1.00^{d}$ & $21.19 \pm 0.98^{d}$ & $20.18 \pm 0.01^{d}$ & $35.17 \pm 0.01^{f}$ & $24.79 \pm 0.03^{d}$ & $35.00 \pm 0.04^{f}$ \\
\hline
\end{tabular}

Values within a column with the same superscript are not significantly different at $P=0.05$; Values are mean $\pm S D, n=5, W A S=$ weeks after sowing, Untreated $=0 \mathrm{~g}$, Treated: $A L_{1}=100 \mathrm{~g}$ agricultural lime, OM $=100 \mathrm{~g}$ organic manure, $A L_{2}=200 \mathrm{~g}$ agricultural lime, $O M_{2}=200 \mathrm{~g}$ organic manure, $O M_{1}+A L_{1}=50 \mathrm{~g}$ organic manure $+50 \mathrm{~g}$ agricultural lime, $O M_{2}+A L_{2}=100 \mathrm{~g}$ organic manure $+100 \mathrm{~g}$ agricultural lime

Table 4. Amelioration effect of soil amendment on leaf fresh weight (g plant ${ }^{-1}$ ) of Vigna unguiculata grown in acidic and alkaline soil

\begin{tabular}{|c|c|c|c|c|c|c|c|c|c|c|}
\hline \multirow{2}{*}{$\begin{array}{l}\text { Weeks } \\
\text { Soil type }\end{array}$} & \multicolumn{2}{|c|}{2 WAS } & \multicolumn{2}{|c|}{4 WAS } & \multicolumn{2}{|c|}{8 WAS } & \multicolumn{2}{|c|}{12 WAS } & \multicolumn{2}{|c|}{16 WAS } \\
\hline & Acidic soil & Alkaline soil & Acidic soil & Alkaline soil & Acidic soil & Alkaline soil & Acidic soil & Alkaline soil & Acidic soil & Alkaline soil \\
\hline Untreated & $1.23 \pm 0.03^{\mathrm{a}}$ & $1.27 \pm 0.03^{\mathrm{a}}$ & $2.47 \pm 0.01^{\mathrm{a}}$ & $3.33 \pm 0.03^{\mathrm{a}}$ & $5.12 \pm 0.02^{\mathrm{a}}$ & $6.20 \pm 0.01^{\mathrm{a}}$ & $8.67 \pm 0.03^{\mathrm{a}}$ & $10.40 \pm 0.08^{\mathrm{a}}$ & $8.66 \pm 0.02^{\mathrm{a}}$ & $10.26 \pm 0.23^{\mathrm{a}}$ \\
\hline \multicolumn{11}{|c|}{ Treated } \\
\hline $\mathrm{AL}_{1}$ & $1.41 \pm 0.02^{b}$ & $1.43 \pm 0.01^{b}$ & $5.23 \pm 0.04^{b}$ & $6.30 \pm 1.00^{b}$ & $12.53 \pm 0.07^{b}$ & $19.10 \pm 1.02^{b}$ & $22.03 \pm 0.02^{b}$ & $25.20 \pm 0.05^{\mathrm{b}}$ & $22.02 \pm 0.02^{b}$ & $25.00 \pm 1.00^{b}$ \\
\hline $\mathrm{AL}_{2}$ & $1.45 \pm 0.05^{\mathrm{bc}}$ & $1.46 \pm 0.04^{b}$ & $2.55 \pm 2 . .^{31 \mathrm{ac}}$ & $6.45 \pm 1.05^{b}$ & $12.97 \pm 0.02^{\mathrm{b}}$ & $19.22 \pm 1.01^{\mathrm{b}}$ & $23.13 \pm 0.01^{\mathrm{c}}$ & $25.43 \pm 0.07^{b}$ & $23.13 \pm 0.98^{\mathrm{c}}$ & $25.06 \pm 0.04^{b}$ \\
\hline $\mathrm{OM}_{1}$ & $1.50 \pm 0.01^{c}$ & $1.61 \pm 0.01^{c}$ & $5.30 \pm 0.06^{c}$ & $6.71 \pm 0.97^{b}$ & $16.25 \pm 0.05^{\mathrm{c}}$ & $26.33 \pm 0.08^{d}$ & $24.47 \pm 0.07^{d}$ & $31.06 \pm 0.02^{d}$ & $24.40 \pm 0.02^{d}$ & $30.92 \pm 0.02^{d}$ \\
\hline $\mathrm{OM}_{2}$ & $1.52 \pm 0.05^{\mathrm{c}}$ & $1.63 \pm 0.03^{c}$ & $6.20 \pm 0.05^{\mathrm{bc}}$ & $8.10 \pm 0.01^{c}$ & $16.17 \pm 0.97^{c}$ & $27.65 \pm 0.05^{\mathrm{e}}$ & $24.67 \pm 0.02^{d}$ & $30.30 \pm 0.10^{c}$ & $24.65 \pm 0.05^{\mathrm{d}}$ & $29.95 \pm 0.02^{\mathrm{c}}$ \\
\hline $\mathrm{AL}_{1}+\mathrm{OM}_{1}$ & $1.62 \pm 0.01^{\mathrm{d}}$ & $2.02 \pm 0.02^{d}$ & $6.31 \pm 1.03^{b c}$ & $9.43 \pm 0.30^{d}$ & $16.46 \pm 0.04^{\mathrm{c}}$ & $25.52 \pm 0.02^{d}$ & $24.51 \pm 1.02^{d}$ & $36.03 \pm 0.03^{f}$ & $24.48 \pm 0.02^{\mathrm{d}}$ & $35.80 \pm 0.03^{f}$ \\
\hline $\mathrm{AL}_{2}+\mathrm{OM}_{2}$ & $1.61 \pm 0.03^{\mathrm{d}}$ & $2.00 \pm 0.01^{d}$ & $6.31 \pm 0.01^{b c}$ & $9.09 \pm 0.01^{\mathrm{cd}}$ & $16.33 \pm 0.03^{c}$ & $24.53 \pm 0.03^{\mathrm{c}}$ & $24.57 \pm 0.03^{d}$ & $33.73 \pm 0.60^{\mathrm{e}}$ & $25.43 \pm 0.19^{\mathrm{e}}$ & $32.37 \pm 0.03^{\mathrm{e}}$ \\
\hline
\end{tabular}
organic manure, $A L_{2}=200 \mathrm{~g}$ agricultural lime, $O M_{2}=200 \mathrm{~g}$ organic manure, $O M_{1}+A L_{1}=50 \mathrm{~g}$ organic manure $+50 \mathrm{~g}$ agricultural lime, OM $+A L_{2}=100 \mathrm{~g}$ organic manure $+100 \mathrm{~g}$ agricultural lime. 
Table 5. Amelioration effect of soil amendment on leaf dry weight (g plant ${ }^{-1}$ ) of Vigna unguiculata grown on acidic and alkaline soil

\begin{tabular}{|c|c|c|c|c|c|c|c|c|c|c|}
\hline \multirow{2}{*}{$\begin{array}{l}\text { Weeks } \\
\text { Soil type }\end{array}$} & \multicolumn{2}{|c|}{2 WAS } & \multicolumn{2}{|c|}{4 WAS } & \multicolumn{2}{|c|}{8 WAS } & \multicolumn{2}{|c|}{12 WAS } & \multicolumn{2}{|c|}{16 WAS } \\
\hline & Acidic soil & Alkaline soil & Acidic soil & Alkaline soil & Acidic soil & Alkaline soil & Acidic soil & Alkaline soil & Acidic soil & Alkaline soil \\
\hline Untreated & $0.24 \pm 0.02^{\mathrm{a}}$ & $0.25 \pm 0.05^{a}$ & $1.56 \pm 0.18^{\mathrm{a}}$ & $1.95 \pm 0.02^{\mathrm{a}}$ & $2.01 \pm 0.01^{\mathrm{a}}$ & $2.87 \pm 0.59^{a}$ & $5.14 \pm 0.01^{\mathrm{a}}$ & $6.71 \pm 0.01^{\mathrm{a}}$ & $5.13 \pm 0.03^{\mathrm{a}}$ & $6.68 \pm 0.02^{\mathrm{a}}$ \\
\hline \multicolumn{11}{|c|}{ Treated } \\
\hline $\mathrm{AL}_{1}$ & $0.24 \pm 0.01$ & $0.25 \pm 0.02^{\mathrm{a}}$ & $3.49 \pm 0.01^{b}$ & $2.26 \pm 0.04^{b}$ & $4.35 \pm 0.01^{\mathrm{b}}$ & $8.60 \pm 0.01^{b}$ & $9.25 \pm 0.01^{b}$ & $16.24 \pm 0.01^{b}$ & $9.20 \pm 0.01^{b}$ & $16.20 \pm 0.02^{b}$ \\
\hline $\mathrm{AL}_{2}$ & $0.25 \pm 0.02^{\mathrm{a}}$ & $0.25 \pm 0.01^{\mathrm{a}}$ & $3.49 \pm 0.03^{b}$ & $2.27 \pm 0.03^{b}$ & $4.37 \pm 0.01^{b}$ & $8.61 \pm 0.01^{b}$ & $9.28 \pm 0.02^{b}$ & $16.26 \pm 0.04^{b}$ & $9.27 \pm 0.01^{\mathrm{c}}$ & $16.21 \pm 0.02^{b}$ \\
\hline $\mathrm{OM}_{1}$ & $0.25 \pm 0.01^{a}$ & $0.26 \pm 0.04^{a}$ & $3.50 \pm 0.02^{b}$ & $3.93 \pm 0.02^{d}$ & $4.76 \pm 0.01^{c}$ & $9.52 \pm 0.03^{c}$ & $9.42 \pm 0.01^{c}$ & $19.61 \pm 0.02^{c}$ & $9.45 \pm 0.05^{d}$ & $19.55 \pm 0.05^{\mathrm{cd}}$ \\
\hline $\mathrm{OM}_{2}$ & $0.26 \pm 0.03^{a}$ & $0.26 \pm 0.01^{\mathrm{a}}$ & $3.72 \pm 0.02^{c}$ & $3.76 \pm 0.06^{c}$ & $4.76 \pm 0.01^{\mathrm{c}}$ & $9.82 \pm 0.02^{c}$ & $9.45 \pm 0.05^{c}$ & $19.63 \pm 0.03^{c}$ & $9.40 \pm 0.01^{d}$ & $19.60 \pm 0.01^{\mathrm{d}}$ \\
\hline $\mathrm{AL}_{1}+\mathrm{OM}_{1}$ & $0.27 \pm 0.01^{\mathrm{a}}$ & $0.37 \pm 0.02^{b}$ & $3.72 \pm 0.02^{c}$ & $4.11 \pm 0.01^{\mathrm{e}}$ & $4.80 \pm 0.03^{d}$ & $10.85 \pm 0.60^{d}$ & $9.43 \pm 0.01^{c}$ & $19.57 \pm 0.02^{\text {cd }}$ & $9.40 \pm 0.02^{d}$ & $19.57 \pm 03^{\mathrm{d}}$ \\
\hline $\mathrm{AL}_{2}+\mathrm{OM}_{2}$ & $0.26 \pm 0.01^{a}$ & $0.37 \pm 0.02^{b}$ & $3.72 \pm 0.02^{c}$ & $4.080 \pm 0.02^{\mathrm{e}}$ & $4.80 \pm 0.02^{d}$ & $10.50 \pm 0.03^{\mathrm{d}}$ & $9.39 \pm 0.07^{c}$ & $19.58 \pm 0.02^{d}$ & $9.40 \pm 0.01^{d}$ & $19.50 \pm 04^{c}$ \\
\hline
\end{tabular}

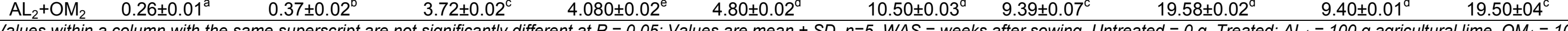

organic manure, $A L_{2}=200 \mathrm{~g}$ agricultural lime, $O M_{2}=200 \mathrm{~g}$ organic manure, $O M_{1}+A L_{1}=50 \mathrm{~g}$ organic manure $+50 \mathrm{~g}$ agricultural lime, $O M_{2}+A L_{2}=100 \mathrm{~g}$ organic manure $+100 \mathrm{~g}$ agricultural lime

Table 6. Amelioration effect of soil amendment on relative growth rate $\left(\mathrm{gg}^{-1} \mathrm{wk}^{-1}\right)$ plant $^{-1}$ of Vigna unguiculata grown in acidic and alkaline soil

\begin{tabular}{|c|c|c|c|c|c|c|c|c|}
\hline \multirow{2}{*}{$\begin{array}{l}\text { Weeks } \\
\text { Soil type }\end{array}$} & \multicolumn{2}{|c|}{4 WAS } & \multicolumn{2}{|c|}{8 WAS } & \multicolumn{2}{|c|}{12 WAS } & \multicolumn{2}{|c|}{16 WAS } \\
\hline & Acidic soil & Alkaline soil & Acidic soil & Alkaline soil & Acidic soil & Alkaline soil & Acidic soil & Alkaline soil \\
\hline Untreated & $0.0071 \pm 0.00^{a}$ & $0.0097 \pm 0.00^{\mathrm{a}}$ & $0.0060 \pm 0.00^{a}$ & $0.0081 \pm 0.00^{\mathrm{a}}$ & $0.0049 \pm 0.00^{a}$ & $0.0048 \pm 0.00^{\mathrm{a}}$ & $0.0033 \pm 0.00^{\mathrm{a}}$ & $0.0048 \pm 0.00^{\mathrm{a}}$ \\
\hline \multicolumn{9}{|c|}{ Treated } \\
\hline $\mathrm{AL}_{1}$ & $0.0101 \pm 0.00^{b}$ & $0.0134 \pm 0.00^{b}$ & $0.0081 \pm 0.00^{b}$ & $0.0105 \pm 0.00^{b}$ & $0.0062 \pm 0.00^{b}$ & $0.0087 \pm 0.00^{\mathrm{bc}}$ & $0.0061 \pm 0.00^{b}$ & $0.0084 \pm 0.00^{b}$ \\
\hline $\mathrm{AL}_{2}$ & $0.0102 \pm 0.00^{b}$ & $0.0139 \pm 0.00^{c}$ & $0.0084 \pm 0.00^{c}$ & $0.0109 \pm 0.00^{c}$ & $0.0062 \pm 0.00^{d}$ & $0.0088 \pm 0.00^{\mathrm{abc}}$ & $0.0061 \pm 0.00^{c}$ & $0.0085 \pm 0.00^{c}$ \\
\hline $\mathrm{OM}_{1}$ & $0.0112 \pm 0.00^{c}$ & $0.0168 \pm 0.00^{d}$ & $0.0092 \pm 0.00^{d}$ & $0.0110 \pm 0.00^{d}$ & $0.0074 \pm 0.00^{\mathrm{e}}$ & $0.0089 \pm 0.00^{b c}$ & $0.0070 \pm 0.00^{C}$ & $0.0085 \pm 0.00^{\mathrm{C}}$ \\
\hline $\mathrm{OM}_{2}$ & $0.0113 \pm 0.00^{c}$ & $0.0172 \pm 0.00^{d}$ & $0.0097 \pm 0.00^{e}$ & $0.0115 \pm 0.00^{\mathrm{e}}$ & $0.0075 \pm 0.00^{\mathrm{ed}}$ & $0.0093 \pm 0.00^{c}$ & $0.0073 \pm 0.00^{c}$ & $0.0090 \pm 0.00^{c}$ \\
\hline $\mathrm{AL}_{1}+\mathrm{OM}_{1}$ & $0.0175 \pm 0.00^{d}$ & $0.0183 \pm 0.00^{\mathrm{e}}$ & $0.0099 \pm 0.00^{\mathrm{e}}$ & $0.0121 \pm 0.00^{f}$ & $0.0071 \pm 0.00^{b}$ & $0.0098 \pm 0.00^{b c}$ & $0.0058 \pm 0.00^{c}$ & $0.0092 \pm 0.00^{d}$ \\
\hline $\mathrm{AL}_{2}+\mathrm{OM}_{2}$ & $0.0180 \pm 0.00^{\mathrm{e}}$ & $0.0186 \pm 0.00^{f}$ & $0.0099 \pm 0.00^{\mathrm{e}}$ & $0.0127 \pm 0.00^{g}$ & $0.0080 \pm 0.00^{c}$ & $0.0098 \pm 0.00^{\mathrm{ab}}$ & $0.0058 \pm 0.00^{c}$ & $0.0095 \pm 0.00^{d}$ \\
\hline
\end{tabular}

organic manure, $A L_{2}=200 \mathrm{~g}$ agricultural lime, $O M_{2}=200 \mathrm{~g}$ organic manure, $O M_{1}+A L_{1}=50 \mathrm{~g}$ organic manure $+50 \mathrm{~g}$ agricultural lime, OM $\mathrm{M}_{2}+A L_{2}=100 \mathrm{~g}$ organic manure $+100 \mathrm{~g}$ agricultural lime 
Table 7. Amelioration effect of soil amendment on net assimilation rate $\left(\mathrm{ggcm}^{-2} \mathrm{wk}^{-1}\right)$ of Vigna unguiculata grown on acidic and alkaline soil

\begin{tabular}{|c|c|c|c|c|c|c|c|c|}
\hline \multirow{2}{*}{$\begin{array}{l}\text { Weeks } \\
\text { Soil type }\end{array}$} & \multicolumn{2}{|c|}{4 WAS } & \multicolumn{2}{|c|}{8 WAS } & \multicolumn{2}{|c|}{12 WAS } & \multicolumn{2}{|c|}{16 WAS } \\
\hline & Acidic soil & Alkaline soil & Acidic soil & Alkaline soil & Acidic soil & Alkaline soil & Acidic soil & Alkaline soil \\
\hline Untreated & $0.0007 \pm 0.00^{a}$ & $0.0009 \pm 0.00^{a}$ & $0.0006 \pm 0.00^{a}$ & $0.0008 \pm 0.00^{\mathrm{a}}$ & $0.0004 \pm 0.00^{\mathrm{a}}$ & $0.0006 \pm 0.00^{a}$ & $0.0004 \pm 0.00^{\mathrm{a}}$ & $0.0005 \pm 0.00^{\mathrm{a}}$ \\
\hline \multicolumn{9}{|c|}{ Treated } \\
\hline $\mathrm{AL}_{1}$ & $0.0010 \pm 0.00^{a}$ & $0.0013 \pm 0.00^{b}$ & $0.0008 \pm 0.00^{b}$ & $0.0012 \pm 0.00^{b}$ & $0.00070 \pm 0.00^{b}$ & $0.0011 \pm 0.00^{b}$ & $0.0007 \pm 0.00^{b}$ & $0.0011 \pm 0.00^{b}$ \\
\hline $\mathrm{AL}_{2}$ & $0.0010 \pm 0.00^{\mathrm{a}}$ & $0.0013 \pm 0.00^{b}$ & $0.0008 \pm 0.00^{b}$ & $0.0012 \pm 0.00^{\mathrm{b}}$ & $0.00070 \pm 0.00^{b}$ & $0.0011 \pm 0.00^{\mathrm{b}}$ & $0.0007 \pm 0.00^{\mathrm{b}}$ & $0.0011 \pm 0.00^{\mathrm{b}}$ \\
\hline $\mathrm{OM}_{1}$ & $0.0010 \pm 0.00^{a}$ & $0.0016 \pm 0.00^{c}$ & $0.0008 \pm 0.00^{b}$ & $0.0014 \pm 0.00^{\mathrm{cd}}$ & $0.00073 \pm 0.00^{b}$ & $0.0012 \pm 0.00^{c}$ & $0.00073 \pm 0.00^{b}$ & $0.0012 \pm 0.00^{c}$ \\
\hline $\mathrm{OM}_{2}$ & $0.0010 \pm 0.00^{a}$ & $0.0016 \pm 0.00^{c}$ & $0.0009 \pm 0.00^{b}$ & $0.0013 \pm 0.00^{c}$ & $0.00075 \pm 0.00^{b}$ & $0.0012 \pm 0.00^{c}$ & $0.00070 \pm 0.00^{b}$ & $0.0012 \pm 0.00^{c}$ \\
\hline $\mathrm{AL}_{1}+\mathrm{OM}_{1}$ & $0.0010 \pm 0.00^{a}$ & $0.0017 \pm 0.00^{d}$ & $0.0009 \pm 0.00^{c}$ & $0.0014 \pm 0.00^{d}$ & $0.00081 \pm 0.00^{c}$ & $0.0012 \pm 0.00^{c}$ & $0.00080 \pm 0.00^{b}$ & $0.0012 \pm 0.00^{c}$ \\
\hline $\mathrm{AL}_{2}+\mathrm{OM}_{2}$ & $0.0010 \pm 0.00^{a}$ & $0.0018 \pm 0.00^{d}$ & $0.0009 \pm 0.00^{c}$ & $0.0014 \pm 0.00^{d}$ & $0.00081 \pm 0.00^{c}$ & $0.0012 \pm 0.00^{c}$ & $0.0080 \pm 0.00^{c}$ & $0.0012 \pm 0.00^{c}$ \\
\hline
\end{tabular}

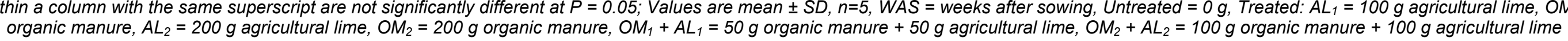

Table 8. Amelioration effect of soil amendment on leaf area ratio $\left(\mathrm{cm}^{-2} \mathrm{~g}^{-1}\right)$ of Vigna unguiculata grown on acidic and alkaline soil

\begin{tabular}{|c|c|c|c|c|c|c|c|c|}
\hline \multirow{2}{*}{$\begin{array}{l}\text { Weeks } \\
\text { Soil type }\end{array}$} & \multicolumn{2}{|c|}{4 WAS } & \multicolumn{2}{|c|}{8 WAS } & \multicolumn{2}{|c|}{12 WAS } & \multicolumn{2}{|c|}{16 WAS } \\
\hline & Acidic soil & Alkaline soil & Acidic soil & Alkaline soil & Acidic soil & Alkaline soil & Acidic soil & Alkaline soil \\
\hline Untreated & $4.47 \pm 0.04^{\mathrm{a}}$ & $5.01 \pm 0.01^{\mathrm{a}}$ & $6.41 \pm 0.05^{\mathrm{a}}$ & $8.71 \pm 0.02^{\mathrm{a}}$ & $8.06 \pm 0.06^{a}$ & $10.08 \pm 0.02^{\mathrm{a}}$ & $8.5 .11 \pm 0.03^{\mathrm{a}}$ & $10.00 \pm 0.02^{\mathrm{a}}$ \\
\hline \multicolumn{9}{|c|}{ Treated } \\
\hline $\mathrm{AL}_{1}$ & $4.66 \pm 0.01^{b}$ & $5.26 \pm 0.04^{b}$ & $8.14 \pm 0.03^{b}$ & $10.59 \pm 0.05^{\mathrm{b}}$ & $9.76 \pm 0.04^{b}$ & $13.18 \pm 0.02^{b}$ & $9.68 \pm 0.04^{\mathrm{b}}$ & $13.13 \pm 0.03^{b}$ \\
\hline $\mathrm{AL}_{2}$ & $4.65 \pm 0.05^{b}$ & $5.20 \pm 0.02^{\mathrm{bc}}$ & $8.17 \pm 0.02^{b}$ & $10.66 \pm 0.01^{b}$ & $9.85 \pm 0.03^{b}$ & $13.11 \pm 0.01^{b}$ & $9.86 \pm 0.02^{\mathrm{bc}}$ & $13.08 \pm 0.02^{b}$ \\
\hline $\mathrm{OM}_{1}$ & $4.74 \pm 0.05^{\mathrm{b}}$ & $5.33 \pm 0.08^{\mathrm{bc}}$ & $8.21 \pm 0.01^{b}$ & $11.17 \pm 0.03^{c}$ & $10.26 \pm 3.50^{c}$ & $13.81 \pm 0.01^{b}$ & $10.24 \pm 0.02^{\mathrm{C}}$ & $13.67 \pm 0.01^{\mathrm{b}}$ \\
\hline $\mathrm{OM}_{2}$ & $4.79 \pm 0.02^{\mathrm{c}}$ & $5.35 \pm 10.00^{\mathrm{bc}}$ & $8.48 \pm 0.01^{b}$ & $11.26 \pm 0.03^{\mathrm{c}}$ & $10.74 \pm 0.04^{c}$ & $14.35 \pm 0.05^{\mathrm{C}}$ & $10.69 \pm 0.01^{\mathrm{C}}$ & $14.28 \pm 0.02^{c}$ \\
\hline $\mathrm{AL}_{1}+\mathrm{OM}_{1}$ & $4.82 \pm 0.06^{c}$ & $5.77 \pm 0.03^{\mathrm{bc}}$ & $8.52 \pm 0.02^{b}$ & $11.35 \pm 0.05^{\mathrm{c}}$ & $10.03 \pm 0.03^{c}$ & $14.34 \pm 0.02^{\mathrm{c}}$ & $10.00 \pm 0.01^{\mathrm{c}}$ & $14.02 \pm 0.02^{c}$ \\
\hline $\mathrm{AL}_{2}+\mathrm{OM}_{2}$ & $4.83 \pm 0.03^{\mathrm{c}}$ & $5.77 \pm 0.04^{\mathrm{bc}}$ & $8.55 \pm 0.03^{b}$ & $11.40 \pm 0.18^{\mathrm{C}}$ & $10.20 \pm 0.10^{c}$ & $14.38 \pm 0.07^{\mathrm{C}}$ & $10.17 \pm 0.02^{\mathrm{C}}$ & $14.30 \pm 0.03^{\mathrm{c}}$ \\
\hline
\end{tabular}
organic manure, $A L_{2}=200 \mathrm{~g}$ agricultural lime, $O M_{2}=200 \mathrm{~g}$ organic manure, $O M_{1}+A L_{1}=50 \mathrm{~g}$ organic manure $+50 \mathrm{~g}$ agricultural lime, OM $\mathrm{M}_{2}+A L_{2}=100 \mathrm{~g}$ organic manure $+100 \mathrm{~g}$ agricultural lime 
Table 9a. Amelioration effect of soil amendment on the yield parameters plant-1 of Vigna unguiculata grown on acidic and alkaline soil

\begin{tabular}{|c|c|c|c|c|c|c|c|c|c|c|}
\hline \multirow{2}{*}{$\begin{array}{l}\text { Yield parameter } \\
\text { Soil type }\end{array}$} & \multicolumn{2}{|c|}{ Pod length (cm) } & \multicolumn{2}{|c|}{ Pod width (mm) } & \multicolumn{2}{|c|}{ Pod weight (g) } & \multicolumn{2}{|c|}{ Number of pods per plant } & \multicolumn{2}{|c|}{ Number of seeds per pod } \\
\hline & Acidic soil & Alkaline soil & Acidic soil & Alkaline soil & Acidic soil & Alkaline soil & Acidic soil & Alkaline soil & Acidic soil & Alkaline soil \\
\hline Untreated & $5.72 \pm 0.02^{\mathrm{a}}$ & $7.03 \pm 0.01^{\mathrm{a}}$ & $0.78 \pm 0.01^{\mathrm{a}}$ & $0.81 \pm 0.01^{\mathrm{a}}$ & $7.11 \pm 0.01^{\mathrm{a}}$ & $8.02 \pm 0.02^{\mathrm{a}}$ & $16.33 \pm 0.01^{\mathrm{a}}$ & $17.46 \pm 0.06^{\mathrm{a}}$ & $4.42 \pm 0.06^{\mathrm{a}}$ & $5.19 \pm 0.01^{\mathrm{a}}$ \\
\hline \multicolumn{11}{|c|}{ Treated } \\
\hline $\mathrm{AL}_{1}$ & $11.94 \pm 0.01^{\mathrm{c}}$ & $14.64 \pm 0.06^{c}$ & $0.82 \pm 0.02^{b}$ & $0.97 \pm 0.01^{b}$ & $7.87 \pm 0.01^{b}$ & $9.45 \pm 0.01^{b}$ & $22.33 \pm 0.14^{b}$ & $25.03 \pm 0.01^{b}$ & $5.36 \pm 0.02^{b}$ & $6.62 \pm 0.02^{b}$ \\
\hline $\mathrm{AL}_{2}$ & $11.81 \pm 0.03^{\mathrm{b}}$ & $14.51 \pm 0.05^{\mathrm{b}}$ & $0.84 \pm 0.04^{b}$ & $0.98 \pm 0.02^{b}$ & $7.80 \pm 0.01^{b}$ & $9.45 \pm 0.01^{\mathrm{b}}$ & $22.43 \pm 0.03^{b}$ & $23.70 \pm 1.16^{\mathrm{c}}$ & $5.39 \pm 0.01^{\mathrm{b}}$ & $6.66 \pm 0.06^{b}$ \\
\hline $\mathrm{OM}_{1}$ & $12.29 \pm 0.01^{\mathrm{e}}$ & $16.29 \pm 0.01^{\mathrm{e}}$ & $0.87 \pm 0.02^{c}$ & $1.01 \pm 0.01^{\mathrm{c}}$ & $7.82 \pm 0.02^{b}$ & $9.51 \pm 0.01^{\mathrm{c}}$ & $23.09 \pm 0.12^{d}$ & $25.67 \pm 0.02^{d}$ & $5.61 \pm 0.01^{\mathrm{c}}$ & $7.17 \pm 0.03^{\mathrm{c}}$ \\
\hline $\mathrm{OM}_{2}$ & $12.24 \pm 0.03^{\mathrm{d}}$ & $16.24 \pm 0.02^{d}$ & $0.87 \pm 0.01^{c}$ & $1.01 \pm 0.01^{\mathrm{c}}$ & $7.83 \pm 0.12^{b}$ & $9.52 \pm 0.02^{c}$ & $22.65 \pm 0.03^{c}$ & $26.62 \pm 0.02^{d}$ & $5.64 \pm 0.01^{\mathrm{C}}$ & $7.20 \pm 0.01^{\mathrm{c}}$ \\
\hline $\mathrm{AL}_{1}+\mathrm{OM}_{1}$ & $13.78 \pm 0.01^{\dagger}$ & $17.01 \pm 0.01^{\dagger}$ & $0.90 \pm 0.01^{\mathrm{d}}$ & $1.03 \pm 0.04^{c}$ & $8.22 \pm 0.01^{\mathrm{c}}$ & $9.55 \pm 0.04^{\mathrm{d}}$ & $23.12 \pm 0.02^{\mathrm{d}}$ & $25.89 \pm 0.01^{\mathrm{d}}$ & $6.02 \pm 0.02^{\mathrm{d}}$ & $8.01 \pm 0.01^{\mathrm{d}}$ \\
\hline $\mathrm{AL}_{2}+\mathrm{OM}_{2}$ & $13.75 \pm 0.05^{\dagger}$ & $17.33 \pm 0.04^{g}$ & $0.90 \pm 0.01^{\mathrm{d}}$ & $1.03 \pm 0.01^{\mathrm{c}}$ & $8.20 \pm 0.03^{c}$ & $9.54 \pm 0.02^{\mathrm{d}}$ & $23.11 \pm 0.11^{\mathrm{d}}$ & $25.94 \pm 0.04^{d}$ & $6.02 \pm 0.02^{\mathrm{d}}$ & $8.07 \pm 0.03^{\mathrm{e}}$ \\
\hline
\end{tabular}
organic manure, $A L_{2}=200 \mathrm{~g}$ agricultural lime, $O M_{2}=200 \mathrm{~g}$ organic manure, $O M_{1}+A L_{1}=50 \mathrm{~g}$ organic manure $+50 \mathrm{~g}$ agricultural lime, OMtre $\mathrm{M}_{2}+A L_{2}=100 \mathrm{~g}$ organic manure $+100 \mathrm{~g}$ agricultural lime

Table 9b. Amelioration effect of soil amendment on the yield parameters plant ${ }^{-1}$ of Vigna unguiculata grown in acidic and alkaline soil

\begin{tabular}{|c|c|c|c|c|c|c|c|c|}
\hline \multirow{2}{*}{$\begin{array}{l}\text { Yield parameters } \\
\text { Soil type }\end{array}$} & \multicolumn{2}{|c|}{ Number of seeds per plant } & \multicolumn{2}{|c|}{ Weight of 100 seeds $(\mathrm{g})$} & \multicolumn{2}{|c|}{ Length of seed (mm) } & \multicolumn{2}{|c|}{ Breadth of seed (mm) } \\
\hline & Acidic soil & Alkaline soil & Acidic soil & Alkaline soil & Acidic soil & Alkaline soil & Acidic soil & Alkaline soil \\
\hline Untreated & $72.18 \pm 0.04^{\mathrm{a}}$ & $90.60 \pm 0.02^{\mathrm{a}}$ & $7.09 \pm 0.02^{\mathrm{a}}$ & $7.87 \pm 0.02^{\mathrm{a}}$ & $0.71 \pm 0.03^{\mathrm{a}}$ & $0.79 \pm 0.02^{\mathrm{a}}$ & $0.64 \pm 0.04^{\mathrm{a}}$ & $0.70 \pm 0.01^{\mathrm{a}}$ \\
\hline \multicolumn{9}{|c|}{ Treated } \\
\hline $\mathrm{AL}_{1}$ & $120.06 \mathrm{E} 2 \pm 0.02^{\mathrm{b}}$ & $165.70 \mathrm{E} 2 \pm 0.02^{b}$ & $8.54 \pm 0.02^{b}$ & $9.34 \pm 0.04^{b}$ & $0.80 \pm 0.02^{b}$ & $0.89 \pm 0.01^{b}$ & $0.6733 \pm 0.06^{b}$ & $0.79 \pm 0.01^{\mathrm{b}}$ \\
\hline $\mathrm{AL}_{2}$ & $120.9 \mathrm{E} 2 \pm 0.03_{\mathrm{c}}$ & $166.70 \mathrm{E} 2 \pm 0.04^{\mathrm{c}}$ & $8.55 \pm 0.01^{\mathrm{b}}$ & $9.34 \pm 0.02^{b}$ & $0.81 \pm 0.03^{\mathrm{b}}$ & $0.90 \pm 0.01^{\mathrm{b}}$ & $0.70 \pm 0.01^{\mathrm{b}}$ & $0.79 \pm 0.01^{\mathrm{b}}$ \\
\hline $\mathrm{OM}_{1}$ & $128.47 \mathrm{E} 2 \pm 1.15^{\mathrm{c}}$ & $183.28 \mathrm{E} 2 \pm 0.02^{\mathrm{d}}$ & $8.57 \pm 0.03^{b}$ & $9.71 \pm 0.01^{c}$ & $0.83 \pm 0.02^{c}$ & $0.90 \pm 0.02^{c}$ & $0.72 \pm 0.02^{b}$ & $0.80 \pm 0.02^{b}$ \\
\hline $\mathrm{OM}_{2}$ & $127.75 \mathrm{E} 2 \pm 0.03^{\mathrm{c}}$ & $191.66 \mathrm{E} 2 \pm 0.03^{\mathrm{e}}$ & $8.57 \pm 0.02^{\mathrm{b}}$ & $9.77 \pm 0.02^{\mathrm{d}}$ & $0.83 \pm 0.02^{c}$ & $0.90 \pm 0.01^{\mathrm{c}}$ & $0.72 \pm 0.01^{\mathrm{b}}$ & $0.84 \pm 0.01^{\mathrm{c}}$ \\
\hline $\mathrm{AL}_{1}+\mathrm{OM}_{1}$ & $139.18 \mathrm{E} 2 \pm 0.02^{\mathrm{e}}$ & $207.38 \mathrm{E} 2 \pm 0.02^{\dagger}$ & $8.83 \pm 0.03^{c}$ & $10.04 \pm 0.04^{\mathrm{e}}$ & $0.83 \pm 0.01^{\mathrm{d}}$ & $0.90 \pm 0.02^{\mathrm{d}}$ & $0.78 \pm 0.02^{c}$ & $0.85 \pm 0.01^{c}$ \\
\hline $\mathrm{AL}_{2}+\mathrm{OM}_{2}$ & $139.12 \mathrm{E} 2 \pm 0.01^{\mathrm{e}}$ & 206.34E $2 \pm 0.04^{\mathrm{g}}$ & $8.88 \pm 0.01^{\mathrm{d}}$ & $10.10 \pm 0.01^{\dagger}$ & $0.83 \pm 0.00^{\mathrm{d}}$ & $0.90 \pm 0.01^{\mathrm{d}}$ & $0.78 \pm 0.02^{\mathrm{c}}$ & $0.85 \pm 0.02^{c}$ \\
\hline
\end{tabular}

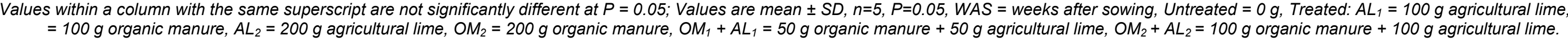


$0.0006 \pm 0.00^{\mathrm{a}}\left(\mathrm{ggcm}^{-2} \mathrm{wk}^{-1}\right)$ was obtained for untreated alkaline soil while highest NAR of $0.0012 \pm 0.00^{c}\left(\mathrm{ggcm}^{-2} \mathrm{wk}^{-1}\right)$ was obtained for alkaline soil treated with $\mathrm{OM}_{1}, \mathrm{OM}_{2}, \mathrm{AL}_{1}+\mathrm{OM}_{1}$ and $\mathrm{AL}_{2}+\mathrm{OM}_{2}$ respectively. This was followed by NAR of $0.0011 \pm 0.00^{b}\left(\mathrm{ggcm}^{-2} \mathrm{wk}^{-1}\right)$ for acidic soil treated with $A L_{1}, A L_{2}$. Net assimilation rate decreased with prolonged period of growth.

\subsection{Amelioration Effect of Soil Amendment on Leaf Area Ratio of Vigna unguiculata Grown on Acidic and Alkaline Soil}

Soil amendment ameliorated the harmful effect of soil $\mathrm{pH}$ on LAR of $V$. unguiculata grown in acidic soil of Akamkpa and alkaline soil of Odukpani with increase in LAR. Untreated acidic and alkaline soil presented the least LAR at 12 WAS of $8.06 \pm 0.06^{\mathrm{a}}$ and $10.08 \pm 0.02^{\mathrm{a}} \mathrm{cm}^{-2} \mathrm{~g}^{-1}$. The highest LAR of $10.74 \pm 0.04^{\mathrm{C}} \mathrm{cm}^{-2} \mathrm{~g}^{-1}$ was obtained for plants grown in acidic soil treated with $\mathrm{OM}_{2}$ at 12 WAS and of $14.38 \pm 0.07^{\mathrm{C}} \mathrm{cm}^{-2} \mathrm{~g}^{-1}$ alkaline soil treated with $\mathrm{AL}_{2}+\mathrm{OM}_{2}$. Corresponding least LAR for untreated soil types were $8.06 \pm 0.06^{\mathrm{a}}$ and $10.08 \pm 0.02^{\mathrm{a}} \mathrm{cm}^{-2} \mathrm{~g}^{-1}$ (Table 8). Unlike RGR and NAR which decreased with increasing period of growth, LAR increased with increasing period of growth.

\subsection{Amelioration Effect of Soil Amendment on the Yield Parameters Per Plant of Vigna unguiculata Grown on Acidic and Alkaline Soil}

The treatment of soil types with soil amendment singly or in combination significantly $(P=0.05)$ increased the yield of $V$. unguiculata. Pod length, pod width, pod weight, number of pods plant $^{-1}$, number of seeds per pod (Table 9a), number of seeds plant $^{-1}$, weight of 100 seeds, length of seed and breadth of seed (Table 9b) were positively impacted on by soil amendment. Yield components Yield was higher for plant grown in alkaline treated and untreated than in acidic soil. Soil amendment led to significant $(P=0.05)$ increase in pod length of the cowpea grown in both soil types. Highest pod length of $13.78 \pm 0.01^{\dagger}$ was found for plant grown in acidic soil treated $\mathrm{AL}_{1}+\mathrm{OM}_{1}$ compared to $5.72 \pm 0.02^{\mathrm{a}}$ $\mathrm{cm}$ for untreated soil. Alkaline soil treated with $\mathrm{AL}_{2}+\mathrm{OM}_{2}$ had pod length of $17.33 \pm 0.04^{\mathrm{g}} \mathrm{cm}$ as against $7.03 \pm 0.01^{\mathrm{a}} \mathrm{cm}$ for untreated soil. Highest pod weight of $0.90 \pm 0.01^{\mathrm{d}}$ and $1.03 \pm 0.01^{\mathrm{c}} \mathrm{g}$ were obtained for acidic and alkaline soils amended with $\mathrm{AL}_{1}+\mathrm{OM}_{1}$ and $\mathrm{AL}_{2}+\mathrm{OM}_{2}$ compared to untreated of $7.11 \pm 0.01^{\mathrm{a}}$ and $8.02 \pm 0.02^{\mathrm{a}} \mathrm{g}$. Number of pods plant $^{-1}$ increased significantly when soils were treated with $\mathrm{OM}$ and $\mathrm{AL}$. Acidic and alkaline soils amended with $\mathrm{AL}_{1}+\mathrm{OM}_{1}$ and $\mathrm{OM}_{2}$ had the highest number of pods plant ${ }^{-1}$ of $23.12 \pm 0.02^{\mathrm{d}}$ and $26.62 \pm 0.02^{\mathrm{d}}$ as against untreated soil values of $16.33 \pm 0.01^{\mathrm{a}}$ and $17.46 \pm 0.06^{\mathrm{a}}$.

Soil amendment increased the number of seeds plant ${ }^{-1}$ to $6.02 \pm 0.02^{\mathrm{d}}$ for plant grown in acid soil amended with $A L_{1}+O M_{1}$ and $\mathrm{AL}_{2}+\mathrm{OM}_{2}$ from $4.42 \pm 0.06^{a}$ untreated, alkaline soil amended with $\mathrm{AL}_{2}+\mathrm{OM}_{2}$ had an increase of $8.07 \pm 0.03^{\mathrm{e}}$ from untreated soil value of $5.19 \pm 0.01^{\mathrm{a}}$ (Table $9 \mathrm{a}$ ).

Number of seeds per pod of $V$. unguiculata grown in soils treated with organic and agricultural lime was higher than that of plants grown in untreated soils. Alkaline soil from Odukpani treated with $\mathrm{AL}_{1}+\mathrm{OM}_{1}$ had the highest number of seeds plant $^{-1}$ followed by $207.38 \mathrm{E} 2 \pm 0.02^{\mathrm{f}}, \quad 191.66 \mathrm{E} 2 \pm 0.03^{\mathrm{e}}$, 183.28E2 $\pm 0.02^{\mathrm{d}}, \quad 166.70 \mathrm{E} 2 \pm 0.04^{\mathrm{c}}$ and 165.70E2 $\pm 0.04^{\mathrm{c}}$ for $\mathrm{AL}_{1}+\mathrm{OM}_{1}, \mathrm{OM}_{2}, \mathrm{OM}_{1}, \mathrm{AL}_{2}$, and $A L_{1}$ respectively compared to untreated soil value of $90.60 \pm 0.02^{\mathrm{a}}$. Acidic soil from Akamkpa amended with $\mathrm{AL}_{1}+\mathrm{OM}_{1}$ had the highest number of seeds plant ${ }^{-1}$ and the least number of seeds for untreated was $72.18 \pm 0.04^{\mathrm{a}}$. A similar trend of higher weight of 100 seeds, length of seed and breadth of seed for soil types treated with soil amendment and untreated soils was found. Soil amendment Weight of 100 seeds length of seed and breadth of seed did not differ significantly between treatments for both soil types but was higher when compared to untreated soils (Table $9 b)$.

\section{DISCUSSION}

The major factor affecting crop productivity is soil $\mathrm{pH}$ which negatively affected the growth of $V$. unguiculata grown in untreated soils as revealed by poor growth of plants. Plant growth components of $V$. unguiculata grown in acidic soil from Akamkpa and alkaline soil from Odukpani were studied with respect to time. At 2 WAS, effect of amelioration on growth parameters though significant was lower when compared to 4 WAP, 8 WAS, 12 WAS and 18 WAS. This was due to poor seedlings establishment at initial period of growth. Growth is a parameter measured by time and two weeks is a small period to allow for meaningful growth in all components studied. Results revealed lower plant height, leaf area, number of leaves 
produced, leaf fresh and weights, RGR, NAR and LAR of $V$. unguiculata caused by soil acidity and alkalinity when compared to plants grown in treated soils. Lower vegetative components have a direct effect on the yield of the cowpea. Similar reports of poor growth and yield of bean varieties grown in untreated soils from three locations compared to the treated are found [9]. Increase in RGR and NAR obtained are in consonance with previous report of soil amendment influence on RGR and NAR [10]. Soil amendment ameliorated soil $\mathrm{pH}$ effect on the growth of $V$. unguiculata as manifested by increase in plant performance. Plants with faster growth rate as promoted by soil amendment in the present research had higher NAR. [16] found that plants with faster growth had a high NAR and plants with high assimilation rates grew faster. Relative growth rate and net assimilation rate decreased steadily with time while the leaf area ratio increased with time for plants grown in treated and untreated soils. There was significant improvement in agronomic components of $V$. unguiculata. The decrease in LAR for the cowpea grown in untreated was accounted for by a reduced portion of leaf weight in the total plant weight due to poor leaf growth occasioned by acidity and alkalinity. Physiologically it could be due to increasing fraction of the assimilate going to the stem. Growth components are indicators of growth and yield. Net assimilation rate is the dry matter increment per unit leaf area per unit time and measures the difference between gross assimilation and respiration. Leaf area ratio is the amount of leaf area which is formed per unit plant weight. So it reflects the influence of the morphology of the plant on its relative growth rate. It should be noted that LAR could be partitioned into specific leaf area (SLA) which is a measure of leaf thickness, and into leaf weight ratio (LWR), which represent that part of total plant weight invested in leaves. Specific leaf weight (SLW) is the inverse of SLA.

Results revealed that $V$. unguiculata grown in untreated soil types had lower yield compared to plants grown in treated soils. The lower yield of $V$. unguiculata was due to low nutrient availability orchestrated by acidity and alkalinity. Similar reports of lower yield of two bean varieties grown in soils of the same location are found [9]. Reduction in the fertility of acidic and alkaline soil is a major limitation for increase in the yield of $V$. unguiculata in Akamkpa and Odukpani Local Government Areas of Cross River State. Significant yield differences between cowpea grown in treated and untreated soils was observed among pod length, number of pods plant $^{-1}$, number of seeds per pod, number of seeds plant ${ }^{-1}$, weight of 100 seeds which are yield indices. Differences were also found in seed length, seed with and pod width. Application of organic and agricultural lime to the two soil types improved growth and yield of the cowpea which could be attributed to improvement in soil nutritional status as well as influence in soil properties such as aeration, water holding capacity, particle size aggregation which contributed to better productivity. Organic manure provides beneficial effects to the treated soil and increase for nutrient availability, which helps to maintain the quality and yield of crops [17]. Organic manure helps in the preservation of natural resources and in the reduction of degradation of ecosystem. Effective amelioration of acidity and alkalinity in this study could also be attributed to good mixture of soil with amendments since the efficacy of soil amendments depend to a large extent on how thoroughly mixed it is with the soil.

Yield components of $V$. unguiculata grown in acidic and alkaline soils of Akamkpa and Odukpani in this study were meaningfully improved upon treatment with soil amendment. Reports on growth and yield improvement of Phaseolus vulgaris and Vigna aconitifolia are found [9]. Managing the harmful effects of soil acidity by liming has showed improvement in crop yield [18]. Application of lime to agricultural acid soils has been in use for many years as amelioration strategy in improving the productivity of crops [19]. Lime is an agricultural material capable of neutralizing soil acidity by increasing soil $\mathrm{pH}$. When $\mathrm{pH}$ falls below 5 , aluminum is released into the soil which was the case with untreated acidic and alkaline soils with attendant effect on crop productivity. Acidity affects the fertility of the soil through nutrients deficiencies of phosphorus, calcium, magnesium and the presence of phytotoxic nutrients such as aluminum and manganese. The reduction in soil fertility occasioned by acidic and alkaline soils manifest as limitations in the production of more food to meet the food need of the populace. The application of limes reduces $\mathrm{AL}$ and $\mathrm{Mn}$ toxicity with the improvement of soil $\mathrm{pH}, \mathrm{Ca}, \mathrm{Mg}$ and increases $\mathrm{P}$ uptake as well as plant rooting system [20]. Lime serve as an important alternative in sustainable soil management for restoring soil fertility. Soil $\mathrm{pH}$ is increased by liming. Correcting soil acidity and alkalinity by the use of lime is fundamental for good soil fertility management. The high performance of $V$. 
unguiculata upon treatment with agricultural lime may be attributed not only to increase in the availability of essential nutrients but also to the addition of $\mathrm{Ca}$ and $\mathrm{Mg}$ which improved soil conditions for the promotion of microbial activities and improvement in soil structure. Plant growth is of paramount ecological significance and highly influenced by soil nutrients which are principally limited for plant use by soil $\mathrm{pH}$. Because soil $\mathrm{pH}$ control many important chemical processes that take place in the soil-plant nutrient availability, it is important to maintain proper $\mathrm{pH}$ levels of soils to enhance $V$. unguiculata performance and yield.

\section{CONCLUSION}

Amelioration effect of soil amendment on growth and yield of $V$. unguiculata grown in acidic and alkaline soils was investigated. All growth parameters (plant height, leaf area, number of leaves produced, leaf fresh, leaf dry weight, relative growth rate, net assimilation rate, leaf area ratio) of $V$. unguiculata grown in acidic and alkaline soils from Akamkpa and Odukpani treated with organic and agricultural lime showed significant improvement compared to plants grown in untreated soils. There was no significant increase in vegetative parameters at 2 WAP, increase however, became obvious with prolonged period of growth. Yield components (pod length, number of pods plant ${ }^{-1}$, number of seeds per pod, number of seeds plant ${ }^{-1}$ weight of 100 seeds and seed breadth) of the cowpea grown in amended soils increased significantly. Results of parameters studied depicted higher growth and yield of plants growth in treated and untreated alkaline soil compared to untreated and treated acidic soil. Organic manure used singly and in combination provided better amelioration effect compared to agricultural line. The study has shown that the low seed yield of $V$. unguiculata grown in acidic soil from Akamkpa and alkaline soil from Odukpani in Southern Cross River State could be increase by the addition of organic manure and agricultural lime either singly or in combination.

\section{ACKNOWLEDGEMENT}

The authors appreciate the department of Botany for the Botanical House used in the study.

\section{COMPETING INTERESTS}

Authors have declared that no competing interests exist.

\section{REFERENCES}

1. Simon T. Breeding cowpea Vigna unguiculata I. Walp for quality traits. Annals of Review and Research. 2018;2(3):555609.

2. Ghady AE, Alkoaiki FN. Examination of protein from common plant leaves for use as human food. American Journal of Applied Sciences. 2010;7(3):323-334.

3. Fatokun CA, Tarawali SA., Singh BB, Kormawa PM, Tamo M. Challenges and opportunities for enhancing sustainable cowpea production. In: Proceedings of the World Cowpea Conference III held at the International Institute of Tropical Agriculture (IITA), Ibadan, Nigeria. 2020;7396.

4. Joshi DKS, Hooda JC, Bhatt BL, Gupta HS. Suppressive effects of composts on soil-borne and foliar diseases of French bean in the field of Western Himalayas. Crop Protection. 2016;28:608-615.

5. Eshett ET, John AIO, Anthony SRJ. Physiocochemical, morphological, and clay mineralogical properties of soils overlying basement complex rocks in Ogoja, northern Cross River State of Nigeria.Soil Science and Plant Nutrition. 1990;36(2):203-214.

DOI: $10.1080 / 00380768.10414985$

6. Attoe EE. Fertility status of soils of the two major cocoa producing Local Government Reas (Etung and Ikom) in Cross River State, Nigeria. Nigerian Journal of Soil Science. 2018;28(2):53-61.

7. Akpan JF, Aki EE, Isong IA. Comparative assessment of wetland and coastalsoils in Calabar, Cross River State. Global Journal of Agricultural Sciences. 2017;16:17-30.

DOI: http://dx.doi.org/10.4314/gjass.v16i1.3

8. Afu SM, Isong IA, Aki EE. Variability of selected physioco-chemical properties of soil overlying different parent materials in Odukpani, Cross River State. International Journal of Plant and Soil Science. 2017;20(6):1-14.

9. Effa EA, Mofunanya AAJ, Ngele BA. Effect of organic manure and agricultural lime on growth and yield of two bean varieties. International Journal of Plant and Soil Science. 2019;30(4):1-18. DOI: 10.9734/IJPSS/2019/v30i430185

10. Effa EA, Mofunanya AAJ, Ngele BA. Influence of soil amendment on the relative growth rate and net assimilation rate of 
Phaseolus vulgaris and Vigna aconitifolia. Journal of Applied Life Sciences International. 2020;22(4):1-13.

DOI: 10.9734/JALSI/2019/v22i430136

11. Association of Official Analytical Chemist (AOAC). Methods of analysis of soils. (12th ed.), USA: AOAC; 1996.

12. Erlacher WA, Oliveira FL, Fialho GS, Silvia DMN, Carvalho AHO. Models for estimating leaf area of yacon. Horticultura Brasileira. 2016;34:422-427. DOI: $10.1590 / S 0102-05362016003019$

13. Hoffmann WA, Poorter H. Avoiding bias in calculations of relative growth rate. Annals of Botany. 2002;90(1):37-42.

14. Heilmeier $H$, Schulze ED, Wale DM. Carbon and nitrogen partitioning in the biennial monocarp Arctium tomentosum mill. Oecologia. 1986;70:466-467.

15. Atwell BJ, Kriedemann PE, Turnbull CGN. Plant in action: In Adaptation in nature, performance in cultivation. South Yarra, Australia: MacMillian Company; 1999. Avaialble:https://doi.org/10.1006/anbo.199 9.0969 .
16. Li X, Schmid B, Wang F, Paine C. Net assimilation rate determines the growth rate of 14 species of subtropical forest trees. Plos One. 2016;11(3): 644.

17. Thy S. and Buntha P. Evaluation of fertilizer of fresh solid manure, composted manure or biodigester effluent for growing Chinese cabbage. $\quad$ AVRDC, Shan hua, Taiwan; 2005.

18. Farhoodi A, Coventry DR. Field crop responses to lime in the mid-north region of South Australia. Field Crops Research. 2008;108:45-53.

19. Scott BJ, Ridley AM, Conyers MK. Management of soil acidity in long-term pastures of south-eastern Australia: A review. Australian Journal of Experimental Agriculture. 2000;40(8):1173-1198.

20. Nduwuremyi A. Soil acidification and lime quality: Sources of acidity, effect on plant nutrients, efficiency of lime and liming requirements. Research \& Review Journal of Agriculture and Allied Sciences. 2013;2(4):26-34.

(c) 2021 Mofunanya et al.; This is an Open Access article distributed under the terms of the Creative Commons Attribution License (http://creativecommons.org/licenses/by/4.0), which permits unrestricted use, distribution, and reproduction in any medium, provided the original work is properly cited.

Peer-review history:

The peer review history for this paper can be accessed here: http://www.sdiarticle4.com/review-history/70709 